\title{
Aspirin-Induced Delayed Urticaria in Children with Kawasaki Disease: A Retrospective Case-Control Study
}

Yao Lin

Yaqi Li

Jingjing $\mathrm{Ma}$

Aijie Li

Yang Liu

Lin Shi (D)

Department of Cardiology, Children's Hospital, Capital Institute of Pediatrics, Beijing, People's Republic of China
Correspondence: Lin Shi

Department of Cardiology, Children's Hospital, Capital Institute of Pediatrics,

No. 2 Yabao Road, Chaoyang District,

Beijing, 100020, People's Republic of

China

Tel +86-13621096102

Email shilinpediatric@outlook.com
Background: Aspirin remains a key component of the standard therapy for Kawasaki disease (KD) in children. Although it is well known that aspirin can cause hypersensitivity such as aspirin-induced urticaria (AIU), AIU in children with KD has not been described.

Methods: A retrospective case-control study was conducted to investigate AIU clinical features, biochemical parameters, treatment and outcomes in children with KD. Furthermore, biomarkers for predicting AIU were explored using the receiver operating characteristic (ROC) curve analysis.

Results: We identified 46 AIU cases with 22 boys and 24 girls during April 2015May 2019. Eighty-nine age-matched KD patients without AIU were randomly chosen as controls. The proportions of children with allergy history and aspirin doses administered in the 2 groups were found not to be significantly different. AIU group had substantially higher baseline C-reactive protein and NT-proBNP levels, and increased neutrophil percent. AIU appeared $6.0(4.0,8.0)$ days after aspirin treatment. Aspirin withdrawal and anti-allergic treatment were applied for AIU, and AIU disappeared in 1-3 days. Baseline NT-proBNP predicted AIU with an AUC of 0.70 (95\% CI [0.60 to 0.79]) for sensitivity and specificity of $72.1 \%$ and $62.5 \%$, respectively, for a cut-off value of $612.9 \mathrm{mg} / \mathrm{L}$. The length of hospital stay for AIU patients was significantly greater compared with controls.

Conclusion: $\mathrm{AIU}$ in $\mathrm{KD}$ children is not related to gender or aspirin dose, and those with AIU have more severe inflammation at admission. Aspirin should be withdrawn for AIU management. Baseline NT-proBNP may serve as a valuable biomarker to predict AIU.

Keywords: Kawasaki disease, aspirin, aspirin-induced urticaria, case-control study

\section{Introduction}

Kawasaki disease (KD), an acute systemic inflammatory vasculitis, often affects children under 5 years old, and patients with KD frequently develop coronary abnormalities, making it the most common cause of acquired heart disease in children in developed countries. ${ }^{1,2}$ A recent systemic review of literature has revealed that children with KD are more likely to develop allergic diseases, such as urticaria during adolescence and adulthood, and a genetic predisposition to allergies is associated with susceptibility to KD. ${ }^{3}$ Aspirin, also known as acetylsalicylic acid (ASA), is a member of nonsteroidal anti-inflammatory drugs (NSAIDs) and a key component of the standard therapy for KD. ${ }^{2}$ ASA combined with human intravenous immunoglobulin (IVIG) significantly reduces inflammation and arterial damage in $\mathrm{KD}^{4,5}$ 
It is well known that NSAIDs can induce a wide array of hypersensitivity reactions that can affect the respiratory (asthma), cutaneous (urticaria and angioedema) and multiple systems (systemic hypersensitivity syndrome, SHS). ${ }^{6-8}$ Aspirin-triggered cutaneous hypersensitivity reactions including aspirin-induced urticaria (AIU) and angioedema in pediatric populations have been documented..$^{9-11}$ However, AIU clinical features, management and outcomes in children with KD have not been described.

\section{Methods}

\section{Study Subjects and Data Collection}

This is a case-control study approved by the Medical Research Review Board of Children's Hospital (SHERLLM2020004), Capital Institute of Pediatrics, Beijing, in accordance with the Declaration of Helsinki, the Code of Ethics of the World Medical Association. The informed consent was waived as the study is retrospective.

The medical records of children with KD (both complete and incomplete) treated at our department from April 2015 to May 2019 were retrospectively reviewed. The demographic data, clinical features, biochemical parameters, treatment and outcomes were retrieved and analyzed. All KD cases and coronary lesions were diagnosed following the criteria of the American Heart Association (AHA). ${ }^{2}$

\section{Aspirin Treatment}

For all patients, ASA was given at $30-50 \mathrm{mg} / \mathrm{kg} /$ day in the acute phase and $3-5 \mathrm{mg} / \mathrm{kg} / \mathrm{day} 3$ days after the patient was afebrile.

\section{Diagnosis of AIU}

AIU was diagnosed based on the following characteristics: 1) AIU appears after resolution of KD rash; 2) AIU disappears rapidly following aspirin withdrawal and antiallergic therapy; 3) AIU causes itch while KD rash does not; and 4) most KD rashes are polymorphous exanthema, some are macular or morbilliform but few are urticariallike. Children with AIU combined with viral infection were excluded. Recurrence of KD was ruled out if fever, bilateral bulbar conjunctival injection and cervical lymphadenopathy did not recur.

\section{Statistical Analysis}

Data normality was determined by the Shapiro-Wilk test. Parametric continuous data were expressed as mean \pm standard deviation (SD) and analyzed by two tailed Student's $t$-test. Nonparametric data were presented as median (Q1, Q3) and analyzed by the Mann-Whitney test. Biomarkers for predicting AIU were assessed using the receiver operating characteristic (ROC) curve analysis. $P<0.05$ was considered statistically significant. All statistical analyses were performed using the GraphPad 8.0 statistics software (GraphPad Software Inc., San Diego, CA, USA).

\section{Results}

Among a total of 1231 children with KD, 48 had AIU with 2 being excluded from this study due to combined viral infection. The remaining 46 AIU cases include 22 males and 24 females, which showed that gender did not play a role in AIU occurrence. Therefore, 89 age-matched KD patients without AIU were randomly chosen for the control group (Table 1). All patients in the two groups had not received ASA prior to this admission. None of the AIU patients had known NSAID allergy history. Body weight and the proportions of children who had known drug and/ or food allergy history in the 2 groups were not significantly different (Table 1).

Blood testing at admission showed that C-reactive protein (CRP) and N-terminal pro b-type natriuretic peptide (NT-proBNP) levels, and the neutrophil percent were significantly higher in the case group compared with the control group, while other parameters were not significantly different (Table 2). Further ROC curve analysis showed that baseline NT-proBNP had the highest value in predicting AIU with an area under curve (AUC) value of 0.70 ( $95 \%$ CI [ 0.60 to 0.79 ]), compared with 0.62 (95\% CI [0.52 to 0.72]) and 0.64 (95\% CI [0.53 to 0.74]) for CRP and neutrophil percent, respectively (Figure 1). With

Table I Demographics, Weight and Allergy History

\begin{tabular}{|l|c|c|c|}
\hline & $\begin{array}{c}\text { Case Group } \\
(\mathbf{n = 4 6 )}\end{array}$ & $\begin{array}{c}\text { Control Group } \\
(\mathbf{n = 8 9})\end{array}$ & p-value \\
\hline Sex (male/female) & $22 / 24$ & $56 / 33$ & 0.09 \\
\hline Age (y) & $1.8(0.8,2.5)$ & $1.9(1.1,3.0)$ & 0.45 \\
\hline Weight (kg) & $11.5(10.0,14.0)$ & $12(10.5,15.0)$ & 0.21 \\
\hline $\begin{array}{l}\text { Drug allergy } \\
\text { history, n (\%) }\end{array}$ & $4(8.7 \%)$ & $10(11.2 \%)$ & 0.87 \\
\hline $\begin{array}{l}\text { Food or other } \\
\text { allergy history, } \\
\mathrm{n} \text { (\%) }\end{array}$ & $2(4.3 \%)$ & $8(9.0 \%)$ & 0.53 \\
\hline
\end{tabular}


Table 2 Blood Testing Results at Admission

\begin{tabular}{|c|c|c|c|}
\hline & Case & Control & $p$-value \\
\hline White blood cells $\left(\times 10^{9} / \mathrm{L}\right)$ & $17.0 \pm 6.3$ & $15.0 \pm 5.3$ & 0.05 \\
\hline Neutrophil percent (\%) & $75.2(62.7,81.2)$ & $65.2(54.3,75.9)$ & 0.01 \\
\hline Monocyte percent (\%) & $5.0(4.0,7.0)$ & $5.6(4.2,7.5)$ & 0.43 \\
\hline Eosinophils percent (\%) & $1.9(0.9,4.2)$ & $2.6(1.2,4.9)$ & 0.16 \\
\hline Hemoglobin $(g / L)$ & $109.5 \pm 12.1$ & $105.2 \pm 12.6$ & 0.06 \\
\hline Platelets $\left(\times 10^{9} / \mathrm{L}\right)$ & $387(305,523)$ & $376(309,566)$ & 0.98 \\
\hline C-reactive protein (mg/L) & $80.4(62.5,121.5)$ & $54.4(30.5,98.5)$ & 0.03 \\
\hline $\operatorname{ESR}(\mathrm{mm} / \mathrm{h})$ & $65.1 \pm 29.1$ & $67.9 \pm 26.3$ & 0.61 \\
\hline TNF- $\alpha(\mathrm{pg} / \mathrm{mL})$ & $20.7(14.4,28.0)$ & $22.2(16.5,40.3)$ & 0.16 \\
\hline NT-BNP (pg/mL) & $1233(449,2344)$ & $481(212,897)$ & $<0.0001$ \\
\hline ALT $(U / L)$ & $28.9(14.7,108.1)$ & $28.9(17.9,76.4)$ & 0.69 \\
\hline AST (U/L) & $29.1(21.0,47.0)$ & $30.6(22.4,62.4)$ & 0.46 \\
\hline Albumin $(g / L)$ & $32.6 \pm 4.9$ & $33.1 \pm 4.9$ & 0.55 \\
\hline $\operatorname{lgE}(\mathrm{IU} / \mathrm{mL})$ & $60.3(24.1,178.8)$ & $74.6(18.1,148.5)$ & 0.80 \\
\hline
\end{tabular}

Abbreviations: ESR, erythrocyte sedimentation rate; TNF, tumor necrosis factor; NT-BNP, N-terminal pro b-type natriuretic peptide; ALT, alanine aminotransferase; AST, aspartate aminotransferase.

a cut-off value of $612.9 \mathrm{mg} / \mathrm{L}$, NT-proBNP predicted AIU with sensitivity and specificity of $72.1 \%$ and $62.5 \%$, respectively.

AIU appeared $6.0(4.0,8.0)$ days after aspirin administration. Prior to AIU onset, the average aspirin dose taken by the control group was $37.5 \pm 4.9 \mathrm{mg} / \mathrm{kg}$, which was not markedly different than $34.7 \pm 4.7 \mathrm{mg} / \mathrm{kg}$ of the case group. Blood cell counts after AIU occurrence revealed that 15 out of 38 AIU cases (39.5\%) had an eosinophil percent $>5 \%$ compared with $7 / 38$ $(18.4 \%)$ at admission $(p<0.05)$, while total blood white cell and lymphocyte counts were within normal ranges. After the onset of AIU, ASA was immediately withdrawn and replaced with clopidogrel, and cetirizine was given in all AIU cases. AIU disappeared in 1-3 days after aspirin withdrawal and anti-allergic treatment.

Forty patients $(87.0 \%)$ in case group and $73(82.0 \%)$ in control group had KD-associated skin rashes that lasted 5.0 $(4.0,6.0)$ and $4.0(3.0,6.0)$ days, respectively $(p=0.42)$ (Table 3). Two patients in the case group, while none in the control group, had Kawasaki disease shock syndrome. There were no significant differences in the fever duration, the number of IVIG-resistance or the number of coronary artery lesion cases between the 2 groups. The length of hospital stay for the case group was significantly greater (Table 3 ).

\section{Discussion}

In the present study, we investigated AIU in children with $\mathrm{KD}$, and reported the following findings: 1) AIU mostly

\section{CRP}

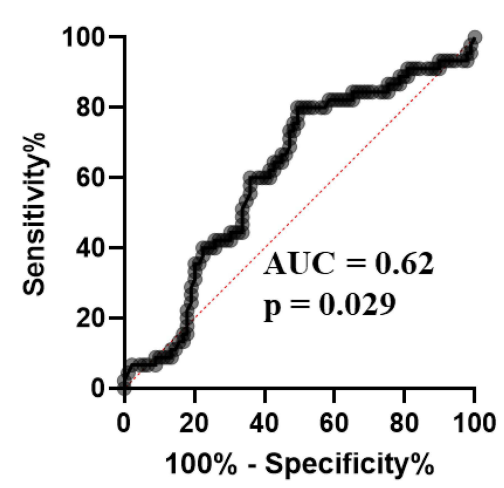

NT-proBNP

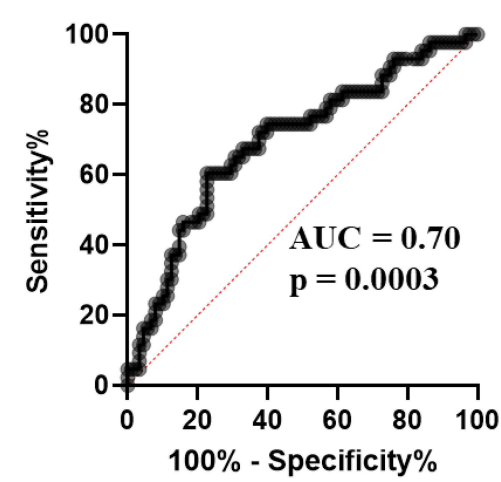

Neutrophil percent

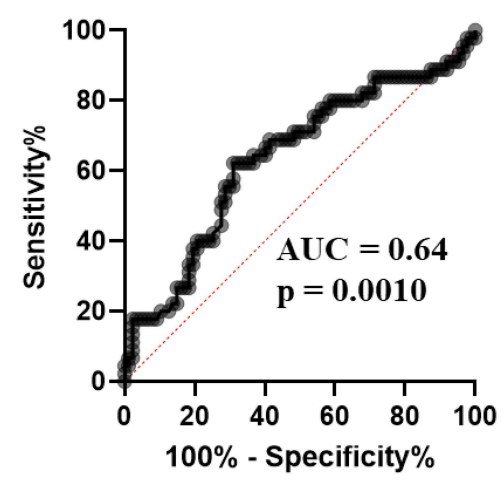

Figure I Assessment of inflammatory parameters as predictors for AIU. The ROC curve analysis revealed that among the 3 significantly increased inflammatory parameters in children with AIU, baseline NT-proBNP had the highest AUC value in predicting AIU. 
Table 3 Clinical Features and the Length of Hospital Stay

\begin{tabular}{|l|c|c|c|}
\hline & Case & Control & p-value \\
\hline KD-associated skin rashes, & $40(87.0 \%)$ & $73(82.0 \%)$ & 0.52 \\
n (\%) & $5.0(4.0,6.0)$ & $4.0(3.0,6.0)$ & 0.42 \\
KD-associated skin rash & & & \\
duration (d) & $7.0(6.0,8.0)$ & $7.0(6.0,8.0)$ & 0.99 \\
Fever duration (d) & $3(6.5 \%)$ & $5(5.6 \%)$ & 1.00 \\
IVIG-resistant cases, n (\%) & $17(37.0 \%)$ & $39(43.8 \%)$ & 0.44 \\
Coronary artery lesions, & & & \\
n (\%) & $10.0(8.0,12.0)$ & $8.0(6.0,9.0)$ & $<0.001$ \\
Length of hospital stay (d) & &
\end{tabular}

Abbreviations: KD, Kawasaki disease; IVIG, intravenous immunoglobulin.

occurred 6 days after ASA administration, and prior to AIU onset, the average aspirin dose administered in the case and control group was not significantly different; 2) at admission the case group had substantially higher levels of CRP and NT-pro BNP, and increased neutrophil counts than the control group, and baseline NT-proBNP had the highest value in predicting AIU; 3) aspirin was replaced with clopidogrel in all AIU patients, and AIU subsided or disappeared 1-3 days later; and 4) The length of hospital stay for patients with AIU was significantly greater.

AIU is one of the most common cutaneous hypersensitivity reactions induced by ASA. ${ }^{9-13}$ It usually develops within 1 hour or up to 24 hours after administration of aspirin. ${ }^{10,12,13}$ However, AIU can also appear 24 hours after drug intake, ${ }^{14-16}$ which is classified as NSAID-induced delayed hypersensitivity reactions (NIDHRs). ${ }^{17}$ A NIDHR is defined as: 1) the reaction is induced by a single NSAID; and 2) it develops more than 24 hours after drug administration. ${ }^{17}$ According to this definition, AIU we described here belongs to the NIDHR. NIDHRs are rare. A case of ASA-induced SHS, a NIDHR, in a child with $\mathrm{KD}$, was previously reported. ${ }^{18}$ Kawakami et al treated a 2-year-old boy who appeared to have recovered without any complications after 1-month of aspirin therapy, but subsequently developed maculopapular eruption and liver dysfunction. ${ }^{18}$ Blood testing showed leukocytosis in the boy, which was not seen in our AIU patients. This discrepancy is probably due to the difference of severity between AIU and SHS. Of note, Kawakami et al observed an abnormally increased count of eosinophils but not lymphocytes in the patient. ${ }^{18}$ Of interest, we also found that AIU caused a significant increase in eosinophilia. These data suggest eosinophilia may be involved in NIDHRs.

Use of moderate- (30-50 mg/kg/day) and high-dose (80$100 \mathrm{mg} / \mathrm{kg} /$ day) aspirin is recommended by the AHA to treat
$\mathrm{KD}$ until the patient is afebrile. ${ }^{2}$ In our practice, we adopted the moderate dosage for the management of KD in the acute phase. In the present study, we did not find significant differences in the average amount of ASA consumed prior to the onset of AIU in the two groups, indicating that ASA dose is not related to AIU. Immediate drug cessation and replacement or desensitization should take place once hypersensitivity to NSAIDs occurs. ${ }^{7}$ For children with KD who are allergic to ASA, replacement of ASA with clopidogrel is recommended by AHA. ${ }^{2}$ In this study, we replaced aspirin with clopidogrel in all patients with AIU.

At the time of admission, children with KD who developed AIU were found to have markedly higher CRP and NTproBNP levels and increased neutrophil percent than controls, suggestive of more severe inflammation in the case group. NT-proBNP is released by cardiomyocytes in response to myocardial wall stress and inflammation, both of which have been found to contribute to higher NT-proBNP levels in patients with KD. ${ }^{19,20}$ As no significant difference was observed in coronary artery lesions between the case and the control group (Table 3), NT-proBNP elevation in the case group was most likely a result of more severe inflammation. We further examined the inflammatory parameters as predictors of AIU, and baseline NT-proBNP was shown to possess the highest value in predicting AIU.

NIDHRs are diagnosed based mainly on clinical history and manifestations as skin patch tests have not been standardized for NIDHR diagnosis. ${ }^{21}$ In this study, all patients with AIU had no known NSAID allergy history, but the withdrawal of aspirin and anti-allergic treatment led to quick subsidence and disappearance of AIU. Recurrence of KD was ruled out in all AIU cases as none of fever, bilateral bulbar conjunctival injection, and cervical lymphadenopathy recurred. Additionally, AIU manifested differently from KD-associated rashes and often caused itching, which aided the diagnosis of AIU in our case series. We observed that children with AIU suffer from the irritating itch caused by AIU, implying the significance of early recognition and prompt management. In the present study, immediate cessation of aspirin intake and anti-allergic therapy were carried out following the guidelines for the diagnosis and management of hypersensitivity to NSAIDs, ${ }^{21}$ which led to satisfactory outcomes.

The mechanism of NIDHRs remains unclear. A link between the leukotriene $\mathrm{C} 4$ synthase (LTC4S) gene polymorphism (A-444C) and aspirin-induced asthma was first identified by Sanak et al. ${ }^{22}$ Later on, a study that evaluated the frequency of the A-444C polymorphism in a Venezuelan 
population discovered that $\mathrm{A}-444 \mathrm{C}$ was related to $\mathrm{AIU}{ }^{23}$ In contrast to these findings, others failed to show A-444C association with aspirin hypersensitivity. ${ }^{24-26}$ This discrepancy could be due to differences in ethnic characteristics, environmental factors and genetic backgrounds. It has been suggested that cell-mediated immune responses take part in NIDHRs, and both CD4+ and CD8+ T lymphocytes are involved in NIDHR pathogenesis. ${ }^{21}$ In the present study, although pathological analysis of skin lesions was not performed, we did not observe abnormal blood lymphocyte counts in AIU children.

Shortcomings of this study include: 1) as this is a retrospective study depending on review of medical records that were originally not designed to collect data for research, some information is bound to be missing, eg, lack of data on lymphocyte subsets, and 2) the sample size is small, limiting the generalization of our findings.

\section{Conclusions}

We report here the largest series of ASA-induced delayed urticaria in pediatric population. AIU in children with KD is not related to gender or aspirin dose, and those with AIU appear to have more severe inflammation at admission. Baseline NT-proBNP may serve as a valuable biomarker to predict AIU. AIU occurs mostly 6 days after aspirin intake, and aspirin should be withdrawn and replaced with clopidogrel for the management of KD. These findings are helpful for clinicians in the early recognition and prompt management of AIU developed in children with $\mathrm{KD}$.

\section{Data Sharing Statement}

Data will be available upon a reasonable request made to the corresponding author.

\section{Ethics Approval and Informed Consent}

This study was approved by the Medical Research Review Board of Children's Hospital (SHERLLM2020004), Capital Institute of Pediatrics, Beijing, China. As this study is retrospective and presents no risk of harm to subjects, and no privacy of individuals is exposed, informed consent was waived. All data presented in the article are de-identified.

\section{Acknowledgments}

We would like to thank Prof. Xiaodai Cui from the Central Laboratory, Children's Hospital, Capital Institute of
Pediatrics, Beijing, China, for technical support. We are also grateful to Mr. Anthony Cannavicci from the University of Toronto, Toronto, Canada, for English editing.

\section{Author Contributions}

All authors made a significant contribution to the work reported, whether that is in the conception, study design, execution, acquisition of data, analysis and interpretation, or in all these areas; took part in drafting, revising or critically reviewing the article; gave final approval of the version to be published; have agreed on the journal to which the article has been submitted; and agree to be accountable for all aspects of the work.

\section{Funding}

No specific funding was received for this study.

\section{Disclosure}

The authors report no conflicts of interest in this work.

\section{References}

1. Dietz SM, van Stijn D, Burgner D, et al. Dissecting Kawasaki disease: a state-of-the-art review. Eur J Pediatr. 2017;176(8):995-1009. doi:10.1007/s00431-017-2937-5

2. McCrindle BW, Rowley AH, Newburger JW, et al. Diagnosis, treatment, and long-term management of Kawasaki disease: a scientific statement for health professionals from the American Heart Association. Circulation. 2017;135(17):e927-e999. doi:10.1161/ CIR.0000000000000484

3. Huang PY, Huang YH, Guo MM, Chang LS, Kuo HC. Kawasaki disease and allergic diseases. Front Pediatr. 2021;8:614386. doi:10.3389/fped.2020.614386

4. Dallaire F, Fortier-Morissette Z, Blais S, et al. Aspirin dose and prevention of coronary abnormalities in Kawasaki disease. Pediatrics. 2017;139(6):e20170098. doi:10.1542/peds.2017-0098

5. Platt B, Belarski E, Manaloor J, et al. Comparison of risk of recrudescent fever in children with Kawasaki disease treated with intravenous immunoglobulin and low-dose vs high-dose aspirin. JAMA Netw Open. 2020;3(1):e1918565. doi:10.1001/jamanetworkopen.20 19.18565

6. Modena B, White AA, Woessner KM. Aspirin and nonsteroidal antiinflammatory drugs hypersensitivity and management. Immunol Allergy Clin North Am. 2017;37(4):727-749.

7. Laidlaw TM, Cahill KN. Current knowledge and management of hypersensitivity to aspirin and NSAIDs. J Allergy Clin Immunol Pract. 2017;5(3):537-545. doi:10.1016/j.jaip.2016.10.021

8. Mourad AA, Bahna SL. Hypersensitivities to non-steroidal anti-inflammatory drugs. Expert Rev Clin Immunol. 2014;10 (9):1263-1268. doi:10.1586/1744666X.2014.946905

9. Cavkaytar O, Arik Yilmaz E, Buyuktiryaki B, Sekerel BE, Sackesen C, Soyer OU. Challenge-proven aspirin hypersensitivity in children with chronic spontaneous urticaria. Allergy. 2015;70 (2):153-160. doi:10.1111/all.12539

10. Kidon MI, Kang LW, Chin CW, et al. Early presentation with angioedema and urticaria in cross-reactive hypersensitivity to nonsteroidal antiinflammatory drugs among young, Asian, atopic children. Pediatrics. 2005;116(5):e675-e680. doi:10.1542/peds.200 5-0969 
11. Wedi B, Kapp A. Aspirin induced adverse skin reactions: new pathophysiological aspects. Thorax. 2000;55(Suppl 2):S70-S71. doi:10.1136/thorax.55.supp1_2.S70

12. Grattan CE. Aspirin sensitivity and urticaria. Clin Exp Dermatol. 2003;28(2):123-127. doi:10.1046/j.1365-2230.2003.01228.x

13. Higashi N, Taniguchi M, Mita H, Higashi A, Akiyama K. Aspirininduced urticaria and angioedema, but not bronchoconstriction, associated with cysteinyl leukotriene overproduction in 2 patients with asthma. J Allergy Clin Immunol. 2002;110(4):666-667. doi:10.1067/ mai.2002.128016

14. Cavkaytar O, Du Toit G, Caimmi D. Characteristics of NSAID-induced hypersensitivity reactions in childhood. Pediatr Allergy Immunol. 2019;30:25-35. doi:10.1111/pai.12980

15. Patel RM, Marfatia YS. Clinical study of cutaneous drug eruptions in 200 patients. Indian J Dermatol Venereol Leprol. 2008;74:430. doi:10.4103/0378-6323.42883

16. Gebhardt M, Wollina U. Cutaneous side-effects of nonsteroidal anti-inflammatory drugs (NSAID). Z Rheumatol. 1995;54:405-412.

17. Kowalski ML, Makowska JS. Seven steps to the diagnosis of NSAIDs hypersensitivity: how to apply a new classification in real practice? Allergy Asthma Immunol Res. 2015;7:312-320. doi:10.4168/aair.2015.7.4.312

18. Kawakami T, Fujita A, Takeuchi S, Muto S, Soma Y. Drug-induced hypersensitivity syndrome: drug reaction with eosinophilia and systemic symptoms (DRESS) syndrome induced by aspirin treatment of Kawasaki disease. J Am Acad Dermatol. 2009;60(1):146-149. doi:10.1016/j.jaad.2008.07.044

19. Dionne A, Dahdah N. A decade of NT-proBNP in acute Kawasaki disease, from physiological response to clinical relevance. Children (Basel). 2018;5(10):E141. doi:10.3390/children5100141
20. Lin KH, Chang SS, Yu CW, et al. Usefulness of natriuretic peptide for the diagnosis of Kawasaki disease: a systematic review and meta-analysis. BMJ Open. 2015;5(4):e006703. doi:10.1136/bmjopen-2014-006703

21. Kowalski ML, Asero R, Bavbek S, et al. Classification and practical approach to the diagnosis and management of hypersensitivity to nonsteroidal anti-inflammatory drugs. Allergy. 2013;68:1219-1232. doi:10.1111/all.12260

22. Sanak M, Simon HU, Szczeklik A. Leukotriene C4 synthase promoter polymorphism and risk of aspirin-induced asthma. Lancet. 1997;350(9091):1599-1600. doi:10.1016/S0140-6736(05)64015-9

23. Sánchez-Borges M, Acevedo N, Vergara C, et al. The A-444C polymorphism in the leukotriene $\mathrm{C} 4$ synthase gene is associated with aspirin-induced urticaria. $J$ Investig Allergol Clin Immunol. 2009;19:375-382.

24. Zhang H, Shi L, Li XH, et al. Gene polymorphism of aspirin-induced urticaria in children with Kawasaki disease. Front Pediatr. 2019;7:505. doi:10.3389/fped.2019.00505

25. Choi JH, Kim SH, Bae JS, et al. Lack of an association between a newly identifi ed promoter polymorphism $(-1702 \mathrm{G}>\mathrm{A})$ of the leukotriene C4 synthase gene and aspirin-intolerant asthma in a Korean population. Tohoku $J$ Exp Med. 2006;208:49-56. doi:10.1620/tjem.208.49

26. Van Sambeek R, Stevenson DD, Baldasaro M, et al. $5^{\prime}$ flanking region polymorphism of the gene encoding leukotriene $\mathrm{C} 4$ synthase does not correlate with the aspirin-intolerant asthma phenotype in the United States. J Allergy Clin Immunol. 2000;106:72-76. doi:10.1067/ mai.2000.107603
International Journal of General Medicine

\section{Publish your work in this journal}

The International Journal of General Medicine is an international, peer-reviewed open-access journal that focuses on general and internal medicine, pathogenesis, epidemiology, diagnosis, monitoring and treatment protocols. The journal is characterized by the rapid reporting of reviews, original research and clinical studies

\section{Dovepress}

across all disease areas. The manuscript management system is completely online and includes a very quick and fair peer-review system, which is all easy to use. Visit http://www.dovepress.com/ testimonials.php to read real quotes from published authors. 